Origin of a localized vibrational mode in a GaSb substrate with a MBE-grown ZnTe epilayer

This content has been downloaded from IOPscience. Please scroll down to see the full text. 2006 Semicond. Sci. Technol. 211224

(http://iopscience.iop.org/0268-1242/21/9/003)

View the table of contents for this issue, or go to the journal homepage for more

Download details:

IP Address: 193.140.249.2

This content was downloaded on 07/10/2016 at 13:42

Please note that terms and conditions apply.

You may also be interested in:

Infrared absorption due to local vibrational modes of nitrogen in GaAs:N and GaAs-baseddilute nitrides

Hans Christian Alt

Sulphur-copper donor-acceptorpairs in GaP

E G Grosche, M J Ashwin, R C Newman et al.

Hydrogen-impurity complexes in III-V semiconductors

W Ulrici

Correlations between $\mathrm{N}-\mathrm{H}$ local vibrational modes in $\mathrm{GaAsN}$ grown by chemical beam epitaxy Kazuma Ikeda, Nobuaki Kojima, Yoshio Ohshita et al.

Influence of surface roughness and interfacial layer on the infrared spectra of V-CVD grown 3C-SiC/Si $\left(\begin{array}{lll}1 & 0 & 0\end{array}\right)$ epilayers

Devki N Talwar, Zhe Chuan Feng, Chee Wee Liu et al.

Splitting of the local mode frequency of substitutional nitrogen

H Ch Alt, Y V Gomeniuk and G Mussler 


\title{
Origin of a localized vibrational mode in a GaSb substrate with a MBE-grown ZnTe epilayer
}

\author{
Hyunjung Kim¹, E Tarhan², G Chen ${ }^{3}$, A K Ramdas 3 , \\ M Dean Sciacca $^{4}$ and R L Gunshor ${ }^{5}$ \\ ${ }^{1}$ Department of Physics and Interdisciplinary Program of Integrated Biotechnology, \\ Sogang University, Seoul 121-742, Korea \\ ${ }^{2}$ Department of Physics, Izmir Institute of Technology, Gulbahcekoyu, Urla, Izmir 35430, \\ Turkey \\ ${ }^{3}$ Department of Physics, Purdue University, West Lafayette, IN 47906-1396, USA \\ ${ }^{4}$ IBM Systems and Technology Group, Hopewell Junction, NY 12533, USA \\ ${ }^{5}$ Department of Electrical and Computer Engineering, Purdue University, West Lafayette, \\ IN 47906-1396, USA \\ E-mail: hkim@sogang.ac.kr
}

Received 8 May 2006, in final form 14 June 2006

Published 18 July 2006

Online at stacks.iop.org/SST/21/1224

\begin{abstract}
A localized vibrational mode (LVM) with a remarkable fine structure is observed in the infrared transmission spectrum of a ZnTe epilayer grown with molecular beam epitaxy (MBE) on a GaSb substrate. On the basis of the $\mathrm{Zn}$ and Te deposited on the GaSb substrate during the MBE growth of $\mathrm{ZnTe}$, and assuming diffusion of $\mathrm{Zn}$ and Te into GaSb, the LVM is attributed to $\mathrm{Zn}$, substitutionally replacing either the cation, $\mathrm{Ga}\left(\mathrm{Zn}_{\mathrm{Ga}}\right)$, or the anion, $\mathrm{Sb}\left(\mathrm{Zn}_{\mathrm{Sb}}\right)$. The frequency of the LVM and its fine structure can then be interpreted in terms of the infrared active modes of ${ }^{64} \mathrm{Zn}$ substituting for $\mathrm{Sb}$ as an anti-site impurity and treating the centre as an $\mathrm{XY}_{4}$ quasimolecule. With $\mathrm{X} \equiv{ }^{64} \mathrm{Zn}$ and $\mathrm{Y} \equiv{ }^{69} \mathrm{Ga}$ and ${ }^{71} \mathrm{Ga}$, occupying the nearest-neighbour sites reflecting all the possible combinations and permutations as well as the natural isotopic abundance of Ga, the fine structure of the LVM can be accounted for quantitatively.
\end{abstract}

(Some figures in this article are in colour only in the electronic version)

\section{Introduction}

With the advent of thin film deposition utilizing molecular beam epitaxy (MBE) and metal organic chemical vapour deposition (MOCVD) nearly 4 decades ago, it became feasible to fabricate epilayers and alternating layers of semiconductors on a suitable substrate. These are of controlled thickness precise on an atomic scale. Thus, superlattices and quantum well structures with atomically smooth interfaces are now routinely produced for optoelectronic and electronic applications. Band offsets between adjacent layers, quantum confinement of electronic levels, excitonic lifetime and the role of zone centre optical phonons in the typical heterostructures of compound semiconductors are illustrative examples of issues which one needs to identify and delineate. Photoluminescence (PL), photoluminescence excitation (PLE), modulated reflectivity and transmission, Raman and Fourier transform spectroscopies are some of the powerful tools exploited in their optical characterization [1].

In the course of the MBE growth of $\mathrm{ZnTe}$ epilayers on GaSb substrates [2,3] and their spectroscopic characterization, we encountered a local vibrational mode (LVM) in GaSb beyond its $\omega_{\mathrm{LO}}$, the high frequency limit of its reststrahlen band. Under the high resolution accessible in Fourier transform spectroscopy, this LVM displays a fascinating fine structure which can be attributed to a substitutional impurity whose vibrations are accompanied by those of its neighbouring atoms [4-6]. In this paper, we present experimental data on 


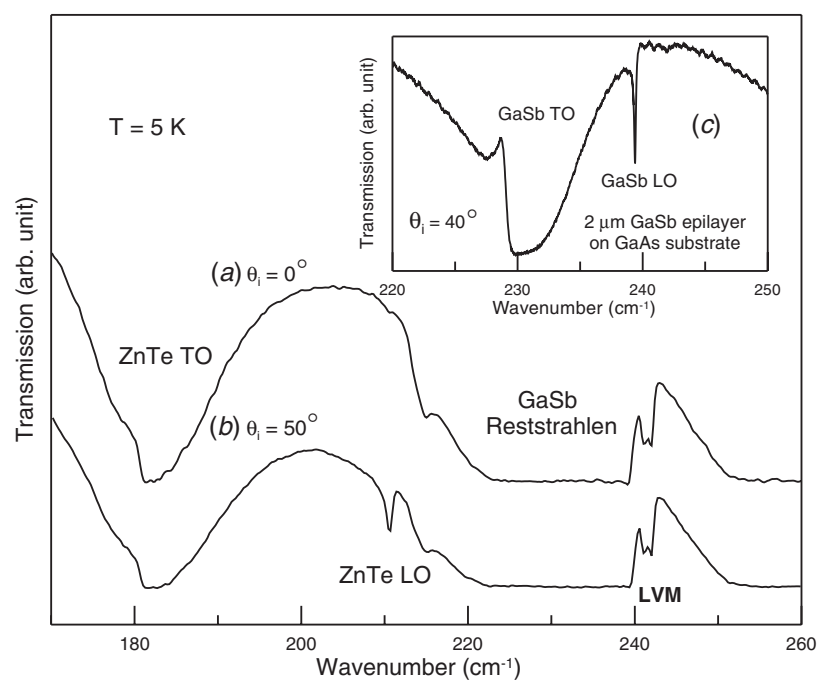

Figure 1. Transmission spectra of $2 \mu \mathrm{m} \mathrm{ZnTe} \mathrm{grown} \mathrm{on} \mathrm{a} \mathrm{GaSb}$ substrate, with the angle of incidence $\left(\theta_{i}\right)$ being $0^{\circ}$ in $(a)$ and $50^{\circ}$ in (b). The spectra were recorded at $5 \mathrm{~K}$ under $0.5 \mathrm{~cm}^{-1}$ resolution. The Berreman geometry, i.e. the oblique incidence $\left(\theta_{i}=50^{\circ}\right)$ in (b) allows the observation of zone centre TO and LO modes of $\mathrm{ZnTe}$. The inset $(c)$ shows the transmission spectrum of a $2 \mu \mathrm{m}$ epilayer of $\mathrm{GaSb}$ on a GaAs substrate for $\theta_{i}=40^{\circ}$, hence permitting the appearance of the zone centre TO and LO GaSb modes. The feature labelled 'LVM' corresponds to a local vibrational mode.

the LVM and interpret its fine structure in terms of the motion of nearest neighbours, taking due account of their isotopic abundances [4].

\section{Experimental procedure}

The procedure for the fabrication of ZnTe epilayers grown directly on nominally 'undoped' p-type GaSb substrates is given in [2,3]. Infrared transmission spectra were recorded with a BOMEM DA $3^{6}$ rapid scanning Fourier transform spectrometer capable of a maximum unapodized resolution of $0.0026 \mathrm{~cm}^{-1}$ and equipped with a globar source, mylar beam splitter, and a composite Si bolometer operated at $4.2 \mathrm{~K}$, incorporating a long-pass cold filter and cone optics. The spectra were recorded at $0.5 \mathrm{~cm}^{-1}$ for low resolution and $0.04 \mathrm{~cm}^{-1}$ for high resolution. The samples were cooled to a temperature of $5 \mathrm{~K}$ in a Janis supervaritemp $10 \mathrm{DT}$ optical cryostat ${ }^{7}$ with polypropylene windows. The sample was intentionally wedged on the back of the substrate in order to prevent channelling in the transmission spectra.

\section{Experimental results and discussion}

The transmission spectrum of a $2 \mu \mathrm{m}$ ZnTe epilayer on the $\mathrm{GaSb}$ substrate is displayed in figure 1( $a$ ) for normal incidence and in figure $1(b)$ for oblique incidence (the angle of incidence, $\left.\theta_{i} \sim 50^{\circ}\right)$ at $0.5 \mathrm{~cm}^{-1}$ resolution. As shown by Berreman [7], for a sufficiently thin film, the minimum in transmission corresponds to $\omega_{\mathrm{TO}}$ in normal incidence whereas for oblique

6 BOMEM Inc., 450 St-Jean Ave., Quebec, Canada, G2E5S5.

7 Janis Research Company, Inc., 2 Jewel Drive, Wilmington, MA 018870896, USA.

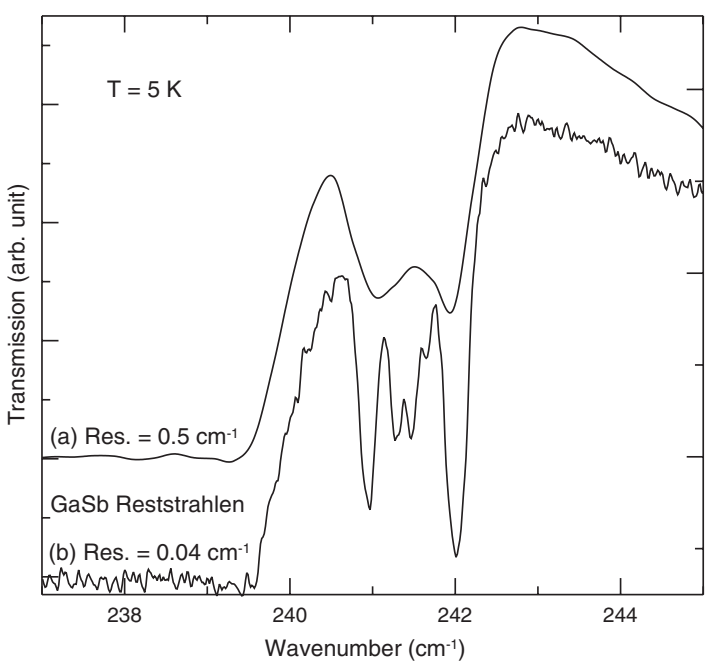

Figure 2. The transmission spectra of the LVM in figures $1(a)$ and $(b)$, recorded at $0.5 \mathrm{~cm}^{-1}$ resolution shown here as $(a)$, compared with that recorded under $0.04 \mathrm{~cm}^{-1}$ resolution in $(b)$.

incidence, such minima occur at $\omega_{\mathrm{TO}}$ as well as at $\omega_{\mathrm{LO}}$, when the thickness $(d)$ of the film satisfies $\left(\omega_{\mathrm{TO}} d / c\right) \ll 1[8]$. The absence of $\omega_{\mathrm{LO}}$ in figure $1(a)$ and its unmistakable appearance in figure $1(b)$ and the presence of $\omega_{\mathrm{TO}}$ in both are the criteria used to identify the TO and LO features of the ZnTe epilayer. Indeed in figure 1 in [9], the authors present a simulation for the Berreman effect in a $2 \mu \mathrm{m}$ thick ZnTe free-standing film which illustrates this identification process. The inset (figure 1(c)) shows the transmission of a $2 \mu \mathrm{m}$ GaSb epilayer on a GaAs substrate recorded in the Berreman geometry, with $\theta_{i}=40^{\circ}$ and $T=5 \mathrm{~K}$; the positions of $\omega_{\mathrm{TO}}$ and $\omega_{\mathrm{LO}}$ are $229.7 \pm$ $0.2 \mathrm{~cm}^{-1}$ and $239.4 \pm 0.05 \mathrm{~cm}^{-1}$, respectively ${ }^{8}$. Hence, the complete opacity in the region marked ' $\mathrm{GaSb}$ reststrahlen' in the transmission spectrum of the GaSb substrate in figures 1(a) and $(b)$ becomes clear.

\subsection{The LVM observed in GaSb}

Beyond the GaSb restrahlen band, there is a transmission window in which the presence of a pair of minima can be clearly noted in figures $1(a)$ and $(b)$ and reproduced in figure 2(a). Under a significantly higher resolution of $0.04 \mathrm{~cm}^{-1}$, the doublet resolves into as many as five transmission minima shown in figure $2(b)$. These spectral features bear a striking similarity to the host isotope fine structure of the LVM of substitutional $\mathrm{C}$ and $\mathrm{Si}$ replacing As in GaAs $[4,5]$. Guided by these observations, we explore the following alternate scenarios: during the MBE growth of ZnTe epilayer on the GaSb substrate, $\mathrm{Zn}$ and Te diffuse into GaSb. $\mathrm{Zn}$ may replace $\mathrm{Ga}$ or $\mathrm{Sb}$ as $\mathrm{Zn}_{\mathrm{Ga}}$ or $\mathrm{Zn}_{\mathrm{Sb}}$ as acceptors, $\mathrm{Zn}_{\mathrm{Sb}}$ being an anti-site substitution. Te may enter as $\mathrm{Te}_{\mathrm{Sb}}$ and $\mathrm{Te}_{\mathrm{Ga}}$ (anti-site) donors. With excess $\mathrm{Te}_{\mathrm{Sb}}$ and $\mathrm{Te}_{\mathrm{Ga}}$, an effective

8 Within experimental errors, our values are in agreement with those reported by Skryabinskii and Ukhanov [10], namely, $\omega_{\mathrm{TO}}=230 \pm 3 \mathrm{~cm}^{-1}$ and $\omega_{\mathrm{LO}}=240 \pm 2 \mathrm{~cm}^{-1}$, measured at $\sim 10 \mathrm{~K}$. Trommer and Ramdas [10] have obtained $\omega_{\mathrm{TO}}=231 \mathrm{~cm}^{-1}$ and $\omega_{\mathrm{LO}}=242 \mathrm{~cm}^{-1}$ at $90 \mathrm{~K}$; these values were deduced from their Raman study of the 'coupled LO-phonon-plasmon modes in $\mathrm{n}-\mathrm{GaSb}$ ' in which the $\omega_{\text {TO }}$ position equals that of the low-frequencycoupled mode at high electron concentration and that of $\omega_{\text {LO }}$ from the Raman signature from the carrier-free depletion layer. 
Table 1. The configurations and combinations of the two gallium or antimony isotopes as they can be arranged in the four equivalent $Y$ positions in the $\mathrm{XY}_{4}$ molecule.

\begin{tabular}{lllllc}
\hline Configuration & $\begin{array}{l}\text { Site } \\
\text { symmetry }\end{array}$ & IR modes & $\begin{array}{l}\text { Distinct } \mathrm{XY}_{4} \\
\text { 'molecules' }\end{array}$ & $\begin{array}{l}\text { Orientational } \\
\text { degeneracy }\end{array}$ & $\begin{array}{l}\text { Total } \\
\text { combinations }\end{array}$ \\
\hline${ }^{M_{i}} \mathrm{Y}_{4}$ & $\mathrm{~T}_{d}$ & $\Gamma_{5}$ & 2 & 1 & 2 \\
${ }^{M_{i}} \mathrm{Y}_{3}^{M_{j}} \mathrm{Y}$ & $\mathrm{C}_{3 v}$ & $\Gamma_{1}+\Gamma_{3}$ & 2 & 4 & 8 \\
${ }^{M_{i}} \mathrm{Y}_{2}^{M_{j}} \mathrm{Y}_{2}$ & $\mathrm{C}_{2 v}$ & $\Gamma_{1}+\Gamma_{2}+\Gamma_{4}$ & 1 & 6 & 6 \\
Total & & 5 & & 16 \\
\hline
\end{tabular}

net compensation (including those of Ga vacancies) occurs, partially suppressing intravalence band transitions of $\mathrm{GaSb}$ [11] and, in turn, opening a region of transparency, an infrared window. Because Te is heavier than both $\mathrm{Ga}$ and $\mathrm{Sb}$, no LVM is to be expected for it. At this stage, one must recognize that $\mathrm{Zn}$ has five stable natural isotopes, namely, ${ }^{64} \mathrm{Zn}(48.6 \%)$, ${ }^{66} \mathrm{Zn}(27.9 \%),{ }^{67} \mathrm{Zn}(4.1 \%),{ }^{68} \mathrm{Zn}(18.8 \%),{ }^{70} \mathrm{Zn}(0.6 \%)$, the members in the parentheses being their natural abundance [12]. The appearance of the doublet under low resolution is shown below to arise from the host isotope fine structure related to the two isotopes of $\mathrm{Sb}$ and $\mathrm{Ga}$ which can occupy the nearest-neighbour positions of $\mathrm{Zn}_{\mathrm{Ga}}$ and $\mathrm{Zn}_{\mathrm{Sb}}$, respectively. If the observed signatures beyond the reststrahlen are due to the ${ }^{64} \mathrm{Zn}$ isotope, one can show that those of ${ }^{66} \mathrm{Zn}$ and the other heavier isotopes will be concealed in the total opacity produced by the reststrahlen band. The presence of an $\mathrm{As}_{\mathrm{Sb}}$ impurity with the nearest neighbours ${ }^{69} \mathrm{Ga}(60.11 \%)$ and ${ }^{71} \mathrm{Ga}$ (39.89\%) can also produce an LVM [13] expected with a not too dissimilar fine structure. However, this has to be excluded on the basis of the growth environments of the MBE facility in which the II-VI and III-V growth chambers are strictly isolated in order to prevent any cross contamination.

\subsection{The origin of the fine structure of the LVM}

In the context of the host isotope fine structure, we proceed to delineate the host isotope effects expected from the ' $\mathrm{XY}{ }_{4}$ ' quasimolecule model. Consider, for example, the $\mathrm{Zn}_{\mathrm{Ga}}$ centre in $\mathrm{GaSb}$, i.e. $\mathrm{X} \equiv \mathrm{Zn}$ and $\mathrm{Y} \equiv \mathrm{Sb}$, with $\mathrm{Zn}$ having a large amplitude and the more massive $\mathrm{Sb}$, anchored to the host lattice displaying a significantly smaller amplitude. As shown in [9], the normal mode of the $\mathrm{XY}_{4}$ molecule relevant in the present context of the LVM is the high frequency, triply degenerate $\Gamma_{5}$ mode, assuming that all Ys belong to the same isotopic species; if they are not, the degeneracy of $\Gamma_{5}$ is lifted to the extent where all the $\mathrm{Y}$ are not identical. The site symmetry of $\mathrm{Zn}_{\mathrm{Ga}}$ is lowered to the trigonal $\mathrm{C}_{3 v}$ if three of the $\mathrm{Y}$ belong to one of the $\mathrm{Sb}$ isotopes and the fourth to the other while it assumes the orthorhombic $\mathrm{C}_{2 v}$ symmetry if two of them belong to one and the other two to the other isotope of Sb. In table 1 we summarize the distinct configurations which result from the occurrence of two $\mathrm{Sb}$ isotopes, ${ }^{121} \mathrm{Sb}$ with $57.3 \%$ natural abundance and ${ }^{123} \mathrm{Sb}$ with $42.7 \%$. On the basis of the natural isotopic abundance of ${ }^{121} \mathrm{Sb}$ and ${ }^{123} \mathrm{Sb}$, we assume that one can attribute the lowest and the highest frequency features in figure 3(a) predominately to the $\Gamma_{5}$ modes of the ${ }^{64} \mathrm{Zn}^{121} \mathrm{Sb}_{4}$ and ${ }^{64} \mathrm{Zn}^{123} \mathrm{Sb}_{4}$ quasimolecules; this identification, together with the frequencies of the two corresponding $\Gamma_{5}$ modes expressed in terms of $\mathrm{M}_{X}, \mathrm{M}_{Y}, \alpha$ and $\beta$ (the atomic masses of $\mathrm{X}$ and $\mathrm{Y}$ and the stretching and bending force constants),
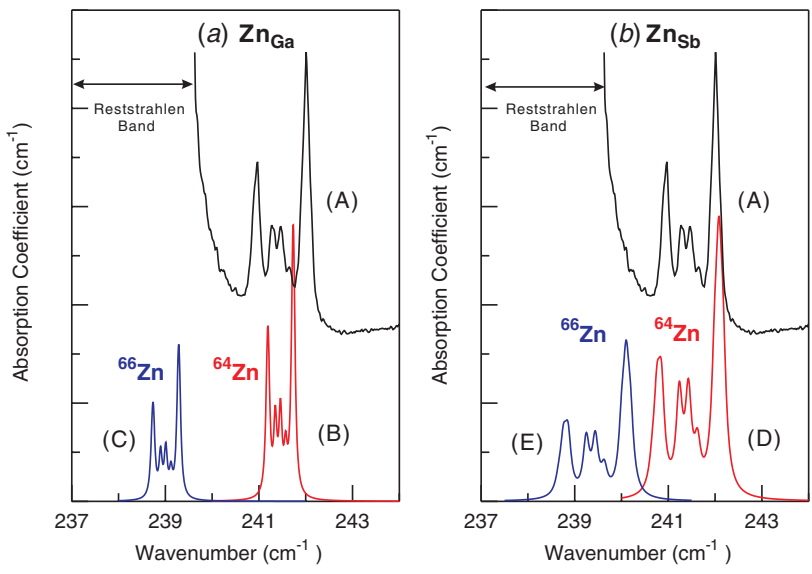

Figure 3. The experimental absorption spectrum of the LVM deduced from the transmission spectrum in figure 2. (a) The experimental absorption spectrum (A) compared with the computed spectra for ${ }^{64} \mathrm{Zn}_{\mathrm{Ga}}(\mathrm{B})$ and ${ }^{66} \mathrm{Zn}_{\mathrm{Ga}}(\mathrm{C})$. (b) The experimental absorption spectrum of the LVM compared with computed spectra for ${ }^{64} \mathrm{Zn}_{\mathrm{Sb}}(\mathrm{D})$ and ${ }^{66} \mathrm{Zn}_{\mathrm{Sb}}(\mathrm{E})$. The rising slope towards lower wavenumbers for the experimental spectrum is due to the onset of the reststrahlen band.

Table 2. Stretching $(\alpha)$ and bending $(\beta)$ force constants which yield the calculated LVM fine structure pattern having the optimum agreement with that displayed by the LVM in figure 2. (The force constants are in the units of $10^{4} \mathrm{dyn}^{-1}$.)

\begin{tabular}{llrl}
\hline Host & Impurity & \multicolumn{1}{l}{$\alpha$} & $\beta$ \\
\hline $\mathrm{GaSb}$ & $\mathrm{Zn}_{\mathrm{Ga}}$ & 97.5 & 4.0 \\
$\mathrm{GaSb}$ & $\mathrm{Zn}_{\mathrm{Sb}}$ & 74.5 & 5.0 \\
$\mathrm{GaSb}$ & $\mathrm{As}_{\mathrm{Ga}}$ & 121.8 & 0.01 \\
$\mathrm{GaSb}$ & $\mathrm{As}_{\mathrm{Sb}}$ & 84.8 & 4.3 \\
\hline
\end{tabular}

allows reasonable estimates of $\alpha$ and $\beta$ as well as the calculation for the modes occurring in between the two extreme frequencies.

In figures 3(a) and $(b)$, the experimental features of the LVM in figure 2 are converted to an absorption coefficient on the basis of the transmission of a semiconductor bilayer (the ZnTe epilayer and the GaSb substrate). The computation of the absorption coefficient due to $\mathrm{ZnTe}$ diffused into $\mathrm{GaSb}$ is carried out following the procedure given by Wemple and Seman $[14]^{9}$. In figure $3(a)$, the computed [6] ${ }^{10}$ absorption spectra for ${ }^{64} \mathrm{Zn}_{\mathrm{Ga}}(\mathrm{B})$ and ${ }^{66} \mathrm{Zn}_{\mathrm{Ga}}(\mathrm{C})$ are compared with the experimental absorption spectrum (A) whereas in figure $3(b)$

9 The refractive indices of GaSb and $\mathrm{ZnTe}$ are 3.8 and 2.7, respectively, deduced from Wemple and Seman's $\epsilon_{\infty}$ given in [15].

${ }^{10}$ The details of the computation procedure for implementing the $\mathrm{XY}_{4}$ model using Mathematica are given in this reference. 

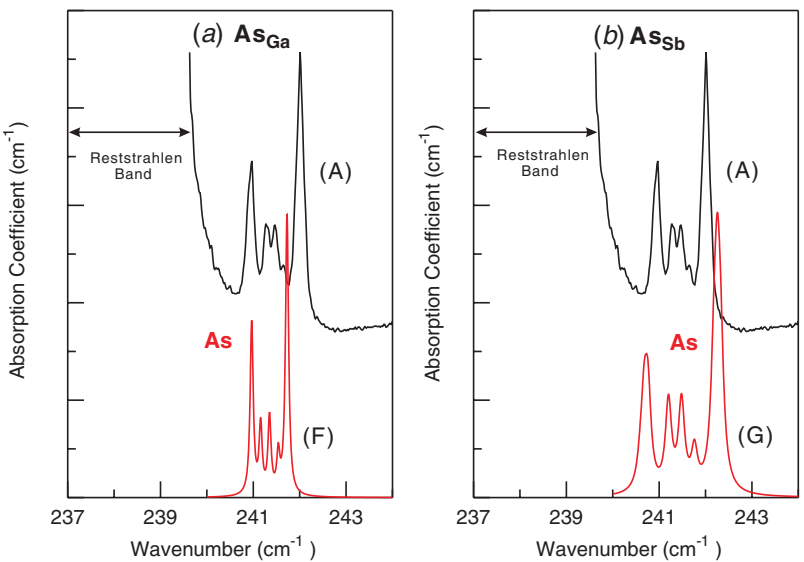

Figure 4. The experimental absorption spectrum of the LVM (A) compared with the computed spectra for $\mathrm{As}_{\mathrm{Ga}}(\mathrm{F})$ in $(a)$ and $\mathrm{As} \mathrm{Sb}$ $(\mathrm{G})$ in $(b)$.

the same comparison is made for ${ }^{64} \mathrm{Zn}_{\mathrm{Sb}}$ (D) and ${ }^{66} \mathrm{Zn}_{\mathrm{Sb}}$ (E). The computed spectra using the stretching $(\alpha)$ and bending $(\beta)$ force constants in table 2 are chosen on the basis of optimum agreement with the experimental absorption maxima. The spectra for ${ }^{66} \mathrm{Zn}$ are also generated with the same force constants as those for ${ }^{64} \mathrm{Zn}$, but with the relative absorption maxima reflecting the ratio of natural abundances for ${ }^{66} \mathrm{Zn} /{ }^{64} \mathrm{Zn}$. As one can see, the overall shape of the fine structure in each case is remarkably similar to that in the experimental spectrum. In figure $3(a)$, the LVMs of ${ }^{66} \mathrm{Zn}$ and the higher atomic mass isotopes of $\mathrm{Zn}$ fall into the reststrahlen of GaSb. However, the spacing between the outermost peaks in the computed spectrum is smaller than that in the experimental absorption spectrum. For $\mathrm{Zn}_{\mathrm{Sb}}$, the anti-site case shown in figure $3(b)$, the spacing between the outermost peaks shows a significantly superior agreement with the experiment. However, in the spectrum for ${ }^{66} \mathrm{Zn}$ computed with the same force constants as those for ${ }^{64} \mathrm{Zn}$ replacing $\mathrm{Sb}$, the highest frequency fine structure component does show some overlap with the experimental absorption spectrum rising towards lower frequencies.

We note here that the outermost peaks in reality consist of three unresolved components corresponding to a superposition of $\Gamma_{1}, \Gamma_{2}$ and $\Gamma_{5}$ modes [16]. The ratio of the computed intensities of the high-frequency outermost peak to that at the lower frequency for ${ }^{64} \mathrm{Zn}_{\mathrm{Sb}}$ and ${ }^{64} \mathrm{Zn}_{\mathrm{Ga}}$ is 1.95 for ${ }^{64} \mathrm{Zn}_{\mathrm{Sb}}$ and 1.6 for ${ }^{64} \mathrm{Zn}_{\mathrm{Ga}}$. Thus, the experimentally observed value of 1.9 for this ratio is yet another confirmation for $\mathrm{Zn}_{\mathrm{Sb}}$ being the correct choice for the impurity site.

Although the presence of As in the GaSb substrate would be inexplicable, given the configuration of the MBE machine, the observed LVM absorption maxima cannot be attributed either to $\mathrm{As}_{\mathrm{Ga}}$ or $\mathrm{As}_{\mathrm{Sb}}$ on the basis of even the best values of $\alpha$ and $\beta$; as can be seen in figure 4 , the spacing of the spectral features just do not match the experimental fine structure.

\section{Concluding remarks}

The unexpected scientific opportunity provided by the occurrence of a LVM in the GaSb substrate with a ZnTe epilayer grown by MBE has led to the discovery of a wellresolved fine structure associated with the nearest-neighbour host atoms of a substitutional impurity. The atomic masses of Te clearly rule out $\mathrm{Te}_{\mathrm{Ga}}$ and $\mathrm{Te}_{\mathrm{Sb}}$ as candidates. The LVM frequency and its host isotope fine structure calculated for both ${ }^{64} \mathrm{Zn}_{\mathrm{Ga}}$ and ${ }^{64} \mathrm{Zn}_{\mathrm{Sb}}$ are compatible with ${ }^{64} \mathrm{Zn}$ as the impurity and the host isotope features consistent with the isotope masses and natural abundance of the nearest-neighbour host atoms, $\mathrm{Sb}$ in the former and $\mathrm{Ga}$ in the latter. The comparison of the line spacings, however, clearly singles out ${ }^{64} \mathrm{Zn}_{\mathrm{Sb}}$, an anti-site impurity, as the substitutional impurity. The appearance of the highest frequency structure in the calculated absorption spectrum between 240 and $241 \mathrm{~cm}^{-1}$ for ${ }^{6} \mathrm{Zn}_{\mathrm{Sb}}$, but not observed in the experiment, could well be due to its close proximity to the reststrahlen. The eigenvectors of the normal modes involving Ga host atoms would presumably interact with the zone centre optical phonons of $\mathrm{GaSb}$ and experience a large broadening [17] ${ }^{11}$. The case of $\mathrm{As}_{\mathrm{Ga}}$ and $\mathrm{As}_{\mathrm{Sb}}$ can be ruled out on the basis of the failure to reproduce the spacing as well as the extremely low likelihood of its presence in the MBE machine whose design strictly separates the III-V and II-VI growth chambers. The impressive similarity of the calculated host isotope fine structure pattern of the LVMs of $\mathrm{Zn}_{\mathrm{Ga}}, \mathrm{Zn}_{\mathrm{Sb}}, \mathrm{As}_{\mathrm{Ga}}, \mathrm{As}_{\mathrm{Sb}}$ and $\mathrm{C}_{\mathrm{As}}$ (in GaAs) as far as their relative intensities, on one hand, and the line spacing unique to each case (the force constants being optimized in the $\mathrm{XY}_{4}$ model) on the other, provide sufficient constraints for the clear selection of $\mathrm{Zn}_{\mathrm{Sb}}$ as the impurity responsible for the experimentally observed LVM. The similarity of the fine structure is an accidental consequence of the existence of two stable isotopes for both $\mathrm{Ga}$ and $\mathrm{Sb}$ and their similar natural isotopic abundance $\left(60.11 \%\right.$ and $39.89 \%$ for ${ }^{69} \mathrm{Ga}$ and ${ }^{71} \mathrm{Ga}$, respectively, as compared to $57.3 \%$ and $42.7 \%$ for ${ }^{121} \mathrm{Sb}$ and ${ }^{123} \mathrm{Sb}$, respectively).

\section{Acknowledgments}

HK acknowledges support from the International Cooperation Research Program of Korean Ministry of Science and Technology (M6-0403-0079) and 2003 Special Research Fund from Sogang University. ET, GC and AKR acknowledge support from the US National Science Foundation (DMR0405082).

\section{References}

[1] Dingle R (ed) 1987 Applications of Multiquantum Wells, Selective Doping, and Superlattices (Semiconductors and Semimetals vol 24) (New York: Academic)

See also Dingle R 1975 Festkorperprobleme Vol XV ed H J Queisser (Nieweg Braunschweig: Pergamon) p 21

[2] Mathine D L et al 1989 Appl. Phys. Lett. 55268

[3] Mathine D L, Durbin S M, Gunshor R L, Kobayashi M, Menke D R, Gonsalves J, Otsuka N, Fu Q, Hagerott M and Nurmikko A V 1990 Surf. Sci. 228344

${ }^{11}$ In the context of the LVMs of GaAs: $\mathrm{C}_{\mathrm{As}}$ and $\mathrm{GaAs}: \mathrm{B}_{\mathrm{As}}$, Leigh et al [17] have considered the influence of the second nearest neighbour and beyond by including anharmonicity. It is of interest to explore if a similar approach would displace and/or broaden the outermost peak of ${ }^{66} \mathrm{Zn}_{\mathrm{Sb}}$ and thus account for its absence in the experimentally observed LVM absorption spectrum of $\mathrm{Zn}_{\mathrm{Sb}}$. Of course, this must occur without producing any disagreement between the computed spectrum for ${ }^{64} \mathrm{Zn}_{\mathrm{Sb}}$ (D) and the experimental (A) spectra in figure $3(b)$. 
[4] Theis W M, Bajaj K K, Litton C W and Spitzer W G 1982 Appl. Phys. Lett. $\mathbf{4 1} 70$

[5] Leigh R S and Newman R C 1982 J. Phys. C: Solid State Phys. 115 L1045

[6] Sciacca M D, Mayur A J, Kim H, Miotkowski I, Ramdas A K and Rodriguez S 1996 Phys. Rev. B 5312878

[7] Berreman D W 1963 Phys. Rev. 1302193

[8] Proix F and Balkanski M 1969 Phys. Status Solidi 32119

[9] Sciacca M D, Mayur A J, Oh E, Ramdas A K, Rodriguez S, Furdyna J K, Melloch M R, Beetz C P and Yoo W S 1995 Phys. Rev. B $\mathbf{5 1} 7744$

[10] Skyrabinskii I V and Ukhanov Yu I 1973 Sov. Phys._Solid State 142838

Trommer R and Ramdas A K 1978 Physics of Semiconductors (Bristol: Institute of Physics Publishing) p 585
[11] Becker W M, Ramdas A K and Fan H Y 1961 J. Appl. Phys. 322094

[12] Emsley J 1998 The Elements 3rd edn (Oxford: Oxford University Press)

[13] Lucovsky G and Chen M F 1970 Solid State Commun. 81397

[14] Wemple S H and Seman J A 1973 Appl. Opt. 122947

[15] Madelung O (ed) 2004 Semiconductors: Data Handbook 3rd edn (Berlin: Springer)

[16] Newman R C 1993 Imperfections in III-V Materials (Semiconductors and Semimetals vol 38) ed E R Weber (San Diego, CA: Academic) chapter 4, p 117

[17] Leigh R S, Newman R C, Sangster M J L, Davidson B R, Ashwin M J and Robbie D A 1994 Semicond. Sci. Technol. 91054 